\title{
Coupling a Surface Acoustic Wave to an Electron Spin in Diamond via a Dark State
}

\author{
D. Andrew Golter, ${ }^{1}$ Thein Oo, ${ }^{1}$ Mayra Amezcua, ${ }^{1}$ Ignas Lekavicius, ${ }^{1}$ Kevin A. Stewart, ${ }^{2}$ and Hailin Wang ${ }^{1}$ \\ ${ }^{1}$ Department of Physics, University of Oregon, Eugene, Oregon 97403, USA \\ ${ }^{2}$ School of Electrical Engineering and Computer Science, \\ Oregon State University, Corvallis, Oregon 97331, USA
}

(Received 2 August 2016; revised manuscript received 28 September 2016; published 20 December 2016)

\begin{abstract}
The emerging field of quantum acoustics explores interactions between acoustic waves and artificial atoms and their applications in quantum information processing. In this experimental study, we demonstrate the coupling between a surface acoustic wave (SAW) and an electron spin in diamond by taking advantage of the strong strain coupling of the excited states of a nitrogen vacancy center while avoiding the short lifetime of these states. The SAW-spin coupling takes place through a $\Lambda$-type three-level system where two ground spin states couple to a common excited state through a phonon-assisted as well as a direct dipole optical transition. Both coherent population trapping and optically driven spin transitions have been realized. The coherent population trapping demonstrates the coupling between a SAW and an electron spin coherence through a dark state. The optically driven spin transitions, which resemble the sideband transitions in a trapped-ion system, can enable the quantum control of both spin and mechanical degrees of freedom and potentially a trapped-ion-like solid-state system for applications in quantum computing. These results establish an experimental platform for spin-based quantum acoustics, bridging the gap between spintronics and quantum acoustics.
\end{abstract}

DOI: 10.1103/PhysRevX.6.041060

\section{INTRODUCTION}

Recent experimental success in coupling a surface acoustic wave (SAW) to a superconducting qubit has led to the emergence of quantum acoustics, the acoustic analog of quantum optics [1]. Acoustic waves propagate at a speed that is 5 orders of magnitude slower than the speed of light, and they couple to artificial atoms through mechanical as well as electromagnetic processes, thereby enabling a new paradigm for on-chip quantum operation and communication. The extensive technologies developed for micro-electromechanical systems (MEMS) can also be adapted for quantum acoustics. Potential applications in quantum information processing, such as phononic cavity QED, mechanically mediated spin entanglement, spin squeezing, and SAW-based universal quantum transducers interfacing a broad array of qubits, have been proposed recently [2-6]. Applications for phonon cooling and lasing have also been considered theoretically $[7,8]$. In addition to superconducting qubits $[1,9,10]$, epitaxially grown as well as gate-defined quantum dots and, more recently, nitrogen vacancy (NV) centers in diamond have also been coupled to mechanical vibrations,

Published by the American Physical Society under the terms of the Creative Commons Attribution 4.0 International license. Further distribution of this work must maintain attribution to the author(s) and the published article's title, journal citation, and DOI.
Subject Areas: Acoustics, Quantum Information, Spintronics including SAWs or mechanical modes of nanomechanical resonators [11-25].

Among the various artificial atoms explored for quantum acoustics, negatively charged NV centers in diamond feature exceptional spin properties, including long decoherence times for electron and nuclear spins and high-fidelity optical state preparation and readout [26-32]. The exquisite quantum control of electron and nuclear spin dynamics in $\mathrm{NV}$ centers has also led to the realization of quantum state transfer between electron and proximal nuclear spins [33]. There is, however, a major technical dilemma. Robust spin qubits necessarily mean extremely weak coupling between the spin and acoustic vibrations [34,35] since otherwise the coupling will lead to rapid decay of the spin coherence. The orbital degrees of freedom associated with the excited states of NV centers can feature much stronger coupling to acoustic vibrations via strain induced by the mechanical vibrations [34-36]. The excited states, however, are not suitable for use as qubits because an electron in these states can quickly decay to the ground spin states through optical spontaneous emission.

This paper reports experimental advances that overcome this dilemma by coupling a SAW to a NV electron spin qubit through an optically prepared dark state. A key ingredient of this process is to use the strain coupling of an excited state to mediate the interaction between the SAW and the NV spin states but without populating the excited state. Our experimental scheme takes advantage of the optically prepared dark state, which traps the electron in the 
ground spin states through quantum interference or through adiabatic evolution of the spin states. In this way, the ground spin states are sensitive to the excited-state strain coupling through optical interactions but are nearly immune to the decay of the excited state.

More specifically, the deformation potential, which characterizes the strength of the excited-state strain coupling, scales with the relevant energy gap and is 5 orders of magnitude greater than the corresponding parameter for the ground-state strain coupling, as shown both theoretically and experimentally $[8,37]$. Optomechanical quantum control of a NV center through the excited-state strain coupling has been demonstrated recently [38]. The strain coupling of the NV excited states has been characterized in detail in a diamond cantilever [37]. Excited-state spin-strain coupling has also been investigated [39].

As illustrated in Fig. 1, the excited-state mediated spinphonon coupling can take place in a $\Lambda$-type three-level system, which features a direct dipole optical transition as well as a phonon-assisted optical transition enabled by the excited-state strain coupling. In this system, the two spin states can couple to the SAW via a dark state, for which the electron is trapped in a special coherent superposition of the two spin states and is thus decoupled from the excited state. Furthermore, in the limit where the external optical fields are sufficiently detuned from the respective dipole optical transitions, the excited state in the $\Lambda$-type three-level system can be adiabatically eliminated from the dynamics of the two spin states. In this case, the optically driven $|e\rangle$

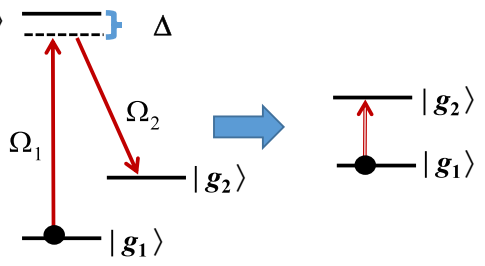

$|e\rangle$

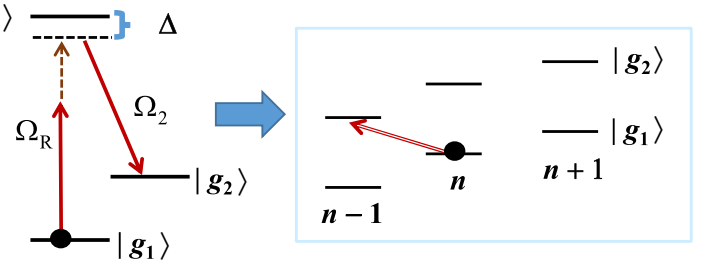

FIG. 1. (a) Schematic of a $\Lambda$-type three-level system driven by two optical fields with respective Rabi frequency, $\Omega_{1}$ and $\Omega_{2}$. In the limit of large dipole detuning, $\Delta$, the system becomes equivalent to an optically driven transition between the two lower states. (b) Schematic of a $\Lambda$-type three-level system driven by an acoustic field (brown dashed line) as well as two optical fields. The $\left|g_{1}\right\rangle$ to $|e\rangle$ transition is a phonon-assisted transition with effective Rabi frequency $\Omega_{R}$. In the limit of large $\Delta$, the system becomes equivalent to an optically driven transition between the phonon ladders of the two lower states, where $n$ denotes the phonon number. transitions take place between the phonon ladders of the two spin states (which we refer to as sideband spin transitions), as illustrated in Fig. 1(b). We have demonstrated the coherent coupling between a SAW and an electron spin in diamond via a dark state by observing phonon-assisted coherent population trapping $(C P T)$. We have also realized optically driven sideband spin transitions in both spectraland time-domain experiments and have shown that these transitions are nuclear spin selective. The single-phonon Rabi frequency for these spin transitions can be 3 orders of magnitude greater than what can be achieved with direct ground-state spin-phonon coupling.

The realization of the phonon-assisted $C P T$, and especially the optically driven sideband spin transitions for a $\mathrm{NV}$ center, establishes an experimental platform for spinbased quantum acoustics. These transitions are analogous to the sideband transitions in the well-known trapped-ion system. As shown for the trapped-ion system, the sideband spin transitions can enable the quantum control of both the spin and mechanical degrees of freedom, leading to one of the most successful platforms for quantum information processing [40-42]. Combined with diamond nanomechanical resonators, which can be fabricated in a diamond-onsilicon or bulk diamond approach [43-47], the excited-state mediated and optically controlled spin-phonon coupling of NV centers opens a promising avenue to realize a solidstate analog of trapped-ion systems.

In the following, we first discuss the experimental setting, including the generation of SAWs and the formation of the relevant $\Lambda$-type three-level systems for a NV center. We then present the experimental demonstration of phonon-assisted $C P T$ and optically driven sideband spin transitions.

\section{EXPERIMENTAL SETTING}

For our experimental studies, a NV center situated a few $\mu \mathrm{m}$ below the diamond surface is subjected to incident laser fields and to a SAW that propagates along and extends approximately one acoustic wavelength below the diamond surface, as illustrated in Fig. 2(a). A confocal optical microscopy setup was used for optical excitation and fluorescence collection of a single NV center [48,49]. The experiments were carried out at $8 \mathrm{~K}$, with the diamond sample mounted in a cold-finger optical cryostat. An offresonant green laser beam $(\lambda=532 \mathrm{~nm})$ was also used to initialize the NV into the $m_{s}=0$ ground state.

\section{A. Generation of surface acoustic waves}

SAWs are widely used in electronic devices, such as MEMS devices. A well-established technique for the generation and detection of SAWs is to pattern interdigital transducers (IDTs) on a piezoelectric substrate. Highfrequency SAW devices have previously been fabricated on diamond [50]. For the samples used in our experiments, 


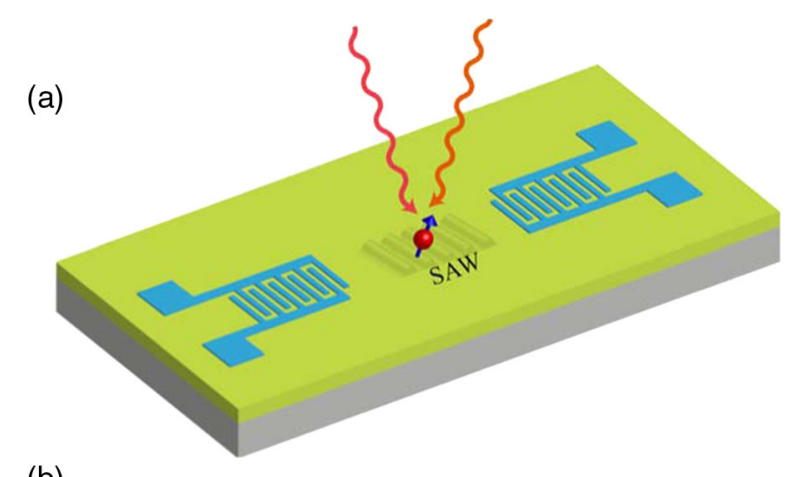

(b)

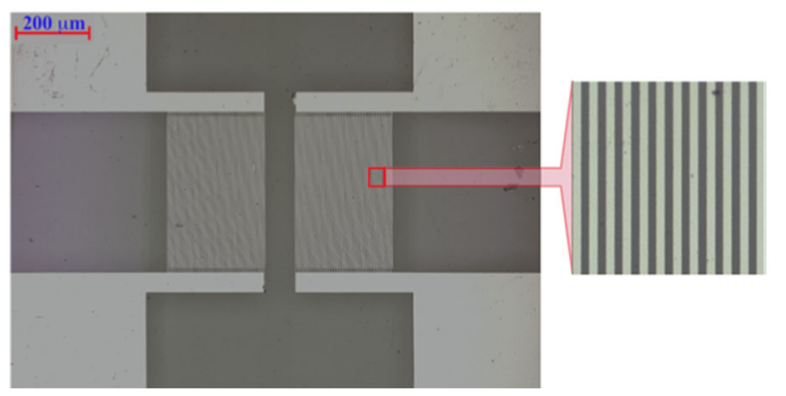

FIG. 2. (a) Schematic of a NV center driven by two optical fields and a SAW field generated by an IDT. (b) Optical image of a pair of IDTs fabricated on the diamond surface.

a 400-nm-thick $\mathrm{ZnO}$ layer, which is piezoelectric, was first sputtered onto the diamond surface. IDTs were then patterned on the $\mathrm{ZnO}$ surface with electron beam lithography. Figure 2(b) shows an optical image of a pair of IDTs, which are $80 \mu \mathrm{m}$ apart, patterned on the $\mathrm{ZnO}$ layer. One IDT, driven by a rf source, serves as a transmitter to generate a SAW via piezoelectric effects. The other, connected to a rf spectrum analyzer, converts the SAW to $r f$ fields and serves as a detector for the characterization of the IDT. Each IDT contains 40 pairs of fingers. The width, $w$, of each finger is $1.5 \mu \mathrm{m}$. The center frequency of the SAW generated is given by $\mathrm{v}_{s} /(4 w)$, where $\mathrm{v}_{s}$ is the SAW velocity. For our structure, the center frequency measured is near $900 \mathrm{MHz}$, yielding a SAW velocity of approximately $5600 \mathrm{~m} / \mathrm{s}$. The electromechanical coupling efficiency of the IDT is estimated to be approximately $0.05 \%$. An input rf power of $1 \mathrm{~W}$ generates a SAW with an amplitude on the order of a pm, as discussed in our earlier work [38].

Since diamond is not piezoelectric and the $\mathrm{ZnO}$ layer deposited on our diamond sample is only $400 \mathrm{~nm}$ in thickness, the SAW reflection from the pair of IDTs is too weak to lead to sharp mechanical resonances, as evidenced by the broad spectral response with a linewidth of about $90 \mathrm{MHz}$ observed for the pair of IDTs. Further improvement in the design and fabrication of the IDT structures is still needed in order to use a pair of IDTs to realize a SAW resonator with a relatively high mechanical $\mathrm{Q}$ factor.

\section{B. $\Lambda$-type three-level systems in a NV center}

In a $\Lambda$-type three-level system, an upper state couples to two lower states through two respective dipole optical transitions [see Fig. 1(a)]. As shown in Fig. 3(a), a NV center features a ground-state spin triplet, with the $m_{s}= \pm 1$ states split from the $m_{s}=0$ state by $2.88 \mathrm{GHz}$, and six excited states, denoted by $A_{1,2}, E_{x, y}$, and $E_{1,2}$ according to their respective symmetry properties [34,35]. Dipole optical transitions in a strain-free NV center occur between the $m_{s}=0$ and the $E_{x, y}$ states and between the $m_{s}= \pm 1$ and the $A_{1,2}$ and $E_{1,2}$ states. With the $m_{s}= \pm 1$ states as the two lower states, a $\Lambda$-type three-level system can be formed through the $A_{2}$ transition (states $A_{1}$ and $E_{1,2}$ can also serve as the upper state, though these states are not as ideal because of various mechanisms of state mixing). In the presence of a dc strain or an external dc electric field, transitions between the $E_{y}$ and the $m_{s}= \pm 1$ states, as well as those between the $E_{1,2}$ and the $m_{s}=0$ states, are allowed because of strain-induced or electric-field-induced state mixing [see Fig. 3(b)] [34,35]. In this case, a $\Lambda$-type three-level system can be formed with the $E_{y}$ state as the upper state and the $m_{s}=0$ state and either of the $m_{s}= \pm 1$ states as the two lower states, as highlighted in Fig. 3(b). CPT and optical spin control have previously been observed in $\Lambda$-type three-level systems with either the $A_{2}$ or $E_{y}$ state as the upper state [48,49,51-53].

For our experimental studies, we have taken advantage of the built-in dc strain in the diamond sample and have used a $\Lambda$-type three-level system with the $E_{y}$ state as the upper state and the $m_{s}=0$ state as one of the lower states. Excited-state mediated spin-phonon coupling can also be implemented with other $\Lambda$-type systems. A technical advantage of using the $m_{s}=0$ state as one of the lower states is that efficient optical spin detection and initialization can be conveniently implemented without the use of microwave fields.

We have carried out detailed photoluminescence excitation experiments to characterize the optical selection rules and to single out the desired $\Lambda$-type three-level system. The inset of Fig. 3(c) shows the fluorescence collected from a single NV center as a function of the frequency of a probe laser near the zero phonon line $(\lambda \sim 637 \mathrm{~nm})$. At each data point, the probe laser is periodically (with a period near $10 \mu \mathrm{s}$ ) alternated with a green laser, which reverses ionization and optical pumping. As a result, the population is initialized into the $m_{s}=0$ ground state. The resulting excitation spectrum features pronounced $E_{x}$ and $E_{y}$ resonances. The splitting between the two resonances is $9.6 \mathrm{GHz}$, indicating a relatively large built-in dc strain [36]. Under this condition, significant strain-induced mixing occurs, which leads to transitions between the $E_{y}$ and the $m_{s}= \pm 1$ states as well as transitions between the $E_{1,2}$ and the $m_{s}=0$ states.

To characterize these processes, we applied a second laser (pump laser), resonant with the $m_{s}=0$ to $E_{x}$ 
(a)

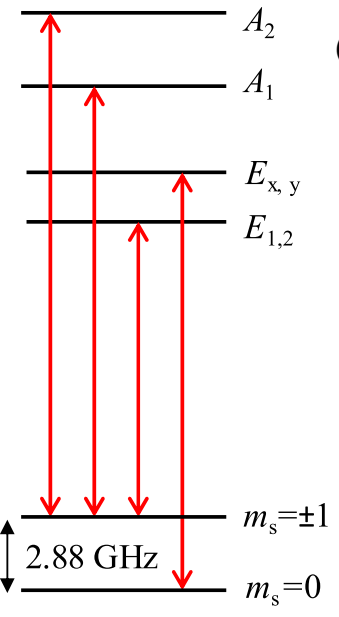

(b)

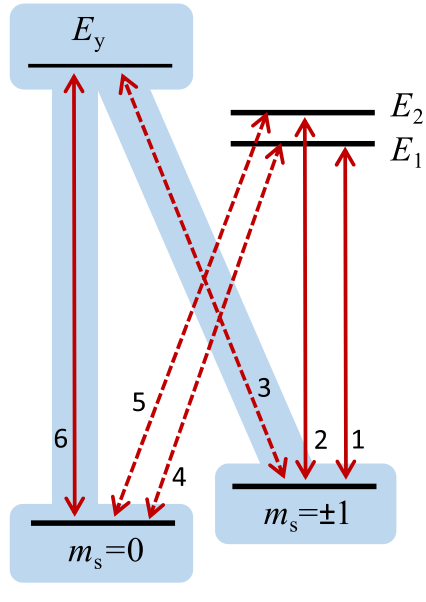

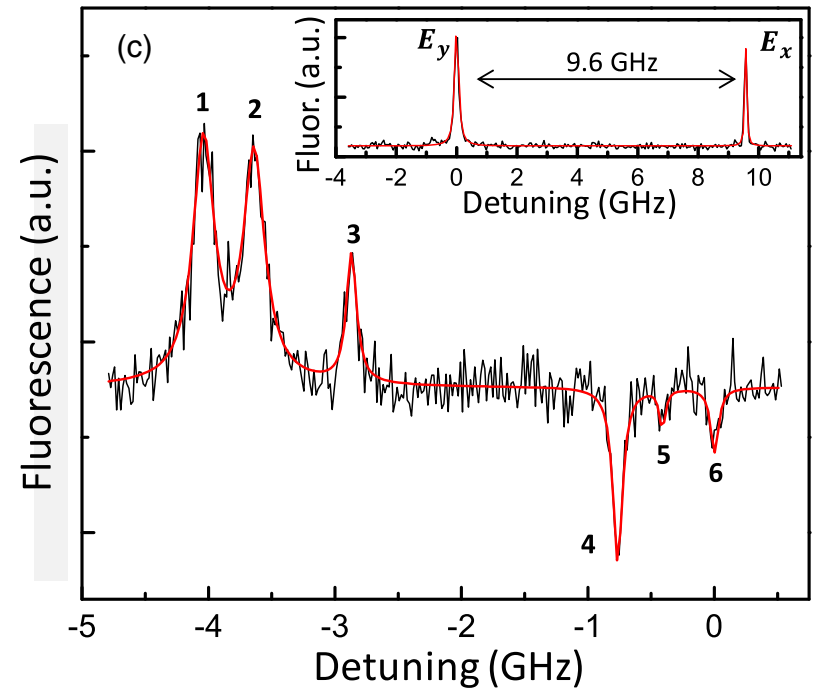

FIG. 3. (a) Energy-level structure and dipole optical transitions of a NV center (strain free). (b) State mixing due to built-in dc strain, leading to additional dipole transitions, as indicated by the dashed lines. Shaded levels and arrows highlight the $\Lambda$-type three-level systems used in our experiments. (c) Excitation spectrum with a pump field fixed at the $E_{x}$ transition. Peak labels indicate the corresponding transitions in (b). Red lines are fits to Lorentzians. Inset: Excitation spectrum obtained with no pump field.

transition, and measured the fluorescence as a function of the probe frequency. The nearly constant fluorescence level in the excitation spectrum shown in Fig. 3(c) is due to the pump-driven $E_{x}$ excitation. This excitation can also pump the electron to the $m_{s}= \pm 1$ ground states since the $E_{x}$ state has a small probability of decaying into the $m_{s}= \pm 1$ states.

The pump-probe excitation spectrum is especially sensitive to the underlying optical pumping process of the probe. As indicated in Fig. 3(c), the probe excitation from the $m_{s}= \pm 1$ ground states leads to three pronounced peaks in the excitation spectrum. These excitations pump the NV back into the $m_{s}=0$ state, increasing the fluorescence from the pump-driven $E_{x}$ transition. In comparison, the

probe excitations from the $m_{s}=0$ state appear as three dips in the excitation spectrum. These excitations pump the NV from the $m_{s}=0$ to the $m_{s}= \pm 1$ states, effectively reducing the fluorescence from the pump-driven $E_{x}$ transition. Because of the efficient optical pumping induced by the probe, transitions from the $m_{s}=0$ to the $E_{1,2}$ states and from the $m_{s}= \pm 1$ to the $E_{y}$ states are more pronounced in the pump-probe excitation spectrum shown in Fig. 3(c) than the probe excitation spectrum shown in the inset of Fig. 3(c). This pump-probe excitation spectrum provides detailed information on the optical transitions of the NV center without the usual application of a microwave field.

\section{PHONON-ASSISTED COHERENT POPULATION TRAPPING}

As discussed in the Introduction, the excited states of NV centers couple strongly to long-wavelength lattice strain. This electron-phonon coupling can lead to a strain-induced energy shift and to state mixing of the relevant excited states [34-36]. For the $E_{y}$ state, the electron-phonon interaction Hamiltonian, describing the strain-induced energy shift, can be written as

$$
H_{e \text {-phonon }}=\hbar g\left(b+b^{+}\right)\left|E_{y}\right\rangle\left\langle E_{y}\right|,
$$

where $b$ is the annihilation operator for the phonon mode, $g=D k_{m} \sqrt{\hbar / 2 m \omega_{m}}$ is the effective electron-phonon coupling rate, $D$ is the deformation potential, $k_{m}$ is the wave number of the phonon mode, $\omega_{m}$ is the phonon frequency, and $m$ is the effective mass of the mechanical resonator. For the phonon-assisted optical transition from the $m_{s}=0$ to the $E_{y}$ state and with a laser field at the red sideband of the optical transition [see Fig. 4(a)], the effective interaction Hamiltonian linear to the mechanical displacement can be derived as (see the Appendix)

$H_{R}=\hbar \frac{\Omega_{0}}{2} \frac{g}{\omega_{m}}\left(b\left|E_{y}\right\rangle\left\langle m_{s}=0\left|+b^{+}\right| m_{s}=0\right\rangle\left\langle E_{y}\right|\right)$,

where $\Omega_{0}$ is the Rabi frequency for the laser field coupling to the transition between the $m_{s}=0$ and the $E_{y}$ state (i.e., the $E_{y}$ transition). The effective Rabi frequency for the phononassisted optical transition (the red sideband transition) is thus given by $\Omega_{R}=g \sqrt{n} \Omega_{0} / \omega_{m}$, where $n$ is the average phonon number. A similar Hamiltonian can also be derived when the laser is at the blue sideband of the optical transition.

We can use the excited-state electron-phonon coupling to mediate the interactions between spin and mechanical degrees of freedom by incorporating a phonon-assisted optical transition into a $\Lambda$-type three-level system, where two ground spin states serve as the two lower states. It is well known that $\Lambda$-type three-level systems feature a dark state, a special coherent superposition of the two lower states, which is decoupled from the upper state [54]. 
(a)
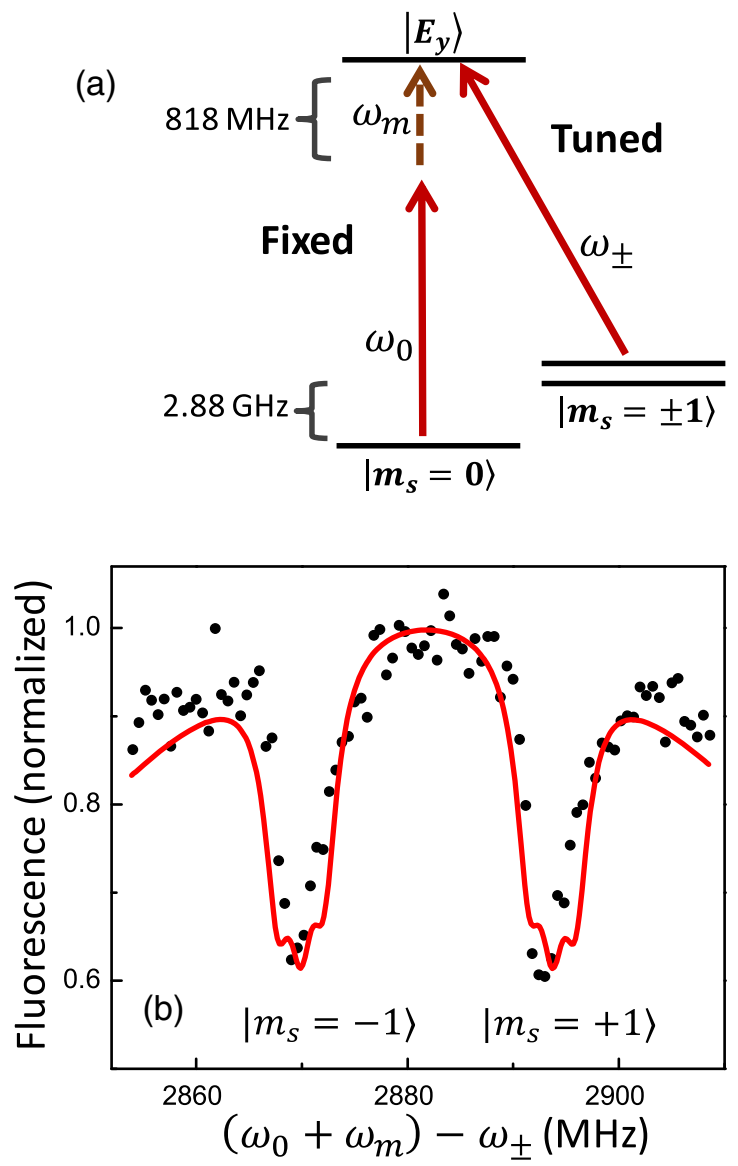

FIG. 4. (a) Energy-level diagram used for phonon-assisted $C P T$. Solid red arrows are the optical fields. The dashed brown arrow is the acoustic field. (b) Fluorescence from state $E_{y}$ as a function of $\omega_{0}+\omega_{m}-\omega_{ \pm}$. The optical power at frequency $\omega_{0}$ and $\omega_{ \pm}$is $4 \mu \mathrm{W}$ and $1 \mu \mathrm{W}$, respectively. The rf input power for the IDT is $0.13 \mathrm{~W}$. The two dips correspond to $\Lambda$-type systems formed with either the $m_{s}=+1$ or the $m_{s}=-1$ state. The solid red curve is the theoretical calculation discussed in the text. The fluorescence is normalized to the peak of the theoretical excitation spectrum with no $C P T$.

This dark state can also be formed for a $\Lambda$-type system that incorporates a phonon-assisted optical transition (see the Appendix). Figure 4(a) shows a $\Lambda$-type three-level system, where the upper state $E_{y}$ couples to states $m_{s}= \pm 1$ through a direct dipole optical transition and to state $m_{s}=0$ through a red sideband transition. For this system, the dark state is given by

$\left|\psi_{d}\right\rangle=\frac{1}{\sqrt{\Omega_{R}^{2}+\Omega_{ \pm}^{2}}}\left(\Omega_{R}\left|m_{s}= \pm 1\right\rangle-\Omega_{ \pm}\left|m_{s}=0\right\rangle\right)$,

where $\Omega_{ \pm}$is the Rabi frequency for the direct dipole optical transition between the $E_{y}$ and the $m_{s}= \pm 1$ states. In this case, the formation of the dark state leads to phonon-assisted $C P T$, with the electron trapped in the two lower states. This dark state can mediate and control the interactions between the spin states and the relevant phonon mode.

For the phonon-assisted $C P T$ experiment, a SAW with $\omega_{m}=818 \mathrm{MHz}$ was coupled to the NV center. The two optical driving fields were derived from a frequencystabilized tunable dye laser. The detuning between the two laser fields was set by two acoustic optical modulators (AOMs). As indicated in Fig. 4(a), one optical field with frequency $\omega_{ \pm}$drives the direct dipole transition between the $E_{y}$ and the $m_{s}= \pm 1$ states. A small magnetic field was also applied to generate a Zeeman splitting, $\omega_{B} / 2 \pi=24 \mathrm{MHz}$, between the $m_{s}= \pm 1$ states. The other optical field with fixed frequency $\omega_{0}$ was tuned to the red sideband of the $E_{y}$ transition such that $\omega_{0}+\omega_{m}$ is resonant with the $E_{y}$ transition. This phonon-assisted optical transition was characterized in detail in our earlier work [38].

A NV center, when pumped into the dark state, will remain trapped in the dark state, resulting in the quenching of the NV fluorescence. Figure 4(b) shows the fluorescence from the $E_{y}$ state as a function of detuning $\omega_{0}+\omega_{m}-\omega_{ \pm}$, for which the NV was initially prepared in the $m_{s}=0$ state and the powers of the two optical fields were adjusted such that $\Omega_{R}$ and $\Omega_{ \pm}$are about equal. Two pronounced dips are observed in Fig. 4(b), when the Raman resonant condition is satisfied, i.e., when $\omega_{0}+\omega_{m}-\omega_{ \pm}$equals the frequency separation between the $m_{s}=0$ and the $m_{s}= \pm 1$ states. Two sets of $\Lambda$-type systems are involved in this case. One contains the $m_{s}=+1$ state and the other the $m_{s}=-1$ state. The two dips correspond to the quenching of the NV fluorescence when the NV is pumped into the respective dark state. These dips are a direct manifestation of the phonon-assisted $C P T$ process, revealing the coherent interaction between the SAW and the relevant electron spin coherence.

We have used the density matrix equations for the $\Lambda$-type three-level system to model the phonon-assisted $C P T$ experiment (see the Appendix). Figure 4(b) shows a general agreement between the experiment and the theory. Note that the $m_{s}= \pm 1$ states exhibit a hyperfine splitting of $2.2 \mathrm{MHz}$ due to coupling with the nitrogen nuclear spin with $I=1$. These hyperfine states, which are included in our model, cannot be clearly resolved in the $C P T$ experiment because of power broadening of the $C P T$ dips and the limited signal-to-noise ratio. With the assumption that $\Omega_{R}=\Omega_{ \pm}$, the observed depth of the CPT dip yields $\Omega_{R} / 2 \pi=8 \mathrm{MHz}$, in agreement with the Rabi frequency estimated from individual dipole optical or phonon-assisted optical transitions [38] and with the Rabi frequency derived from the sideband spin transition experiment, which will be discussed in the next section.

\section{OPTICALLY DRIVEN SIDEBAND SPIN TRANSITIONS}

While the phonon-assisted $C P T$ discussed above demonstrates the coherent coupling between a SAW and an electron spin via a dark state, the residual excitation and 
subsequent decay of the upper state in the $\Lambda$-type system introduces an optically induced decoherence to the spinphonon system. The upper-state excitation, however, can be avoided or reduced through adiabatic passage [55], which has been demonstrated for NV centers [48,56]. For a conventional $\Lambda$-type system, the upper state can also be eliminated adiabatically from the dynamics of the two lower states, if the two optical driving fields are sufficiently detuned from the respective dipole optical transitions. In this adiabatic limit, the three-level system becomes equivalent to an optically driven spin transition between the two lower states, with an effective Rabi frequency for the spin transition given by $\Omega_{S}=\Omega_{1} \Omega_{2} /(2|\Delta|)$, where $\Omega_{1}$ and $\Omega_{2}$ are the Rabi frequencies for the two dipole transitions and $\Delta$ is the dipole detuning, as illustrated in Fig. 1(a)[48]. In analogy, for a $\Lambda$-type three-level system coupling to an acoustic as well as two optical fields and with a sufficiently large detuning for both the optical and the phonon-assisted optical transitions, the three-level system becomes equivalent to optically driven spin transitions between the phonon ladders of the two lower states, with an effective Rabi frequency for the sideband spin transition given by

$$
\Omega_{\mathrm{SS}}=\frac{\Omega_{R} \Omega_{ \pm}}{2|\Delta|}=\frac{\Omega_{0} \Omega_{ \pm}}{2|\Delta| \omega_{m}} g \sqrt{n}=g_{s s} \sqrt{n},
$$

as illustrated in Fig. 1(b). Here, $g_{s s}$ is the single-phonon Rabi frequency for the sideband spin transition.

We have carried out both spectral- and time-domain experiments to demonstrate and characterize the sideband spin transitions. For these experiments, optical driving fields similar to those used for the phonon-assisted $C P T$ experiment were used, except that we set $\Delta / 2 \pi=100 \mathrm{MHz}$. A relatively small detuning was employed such that effects of the upper-state excitation can still be investigated. The optical and rf powers used for Figs. 5 and 6 are the same as those used for Fig. 4. To detect the spin population in the $m_{s}= \pm 1$ states, we used a resonant laser field to excite the NV from the $m_{s}= \pm 1$ states to the $A_{2}$ state and measure the corresponding fluorescence, as illustrated in Fig. 5(a).

\section{A. Spectral-domain experiment}

The pulse sequence used for the spectral-domain experiment is shown in Fig. 5(b). After the initialization of the NV center into the $m_{s}=0$ ground state with a $532-\mathrm{nm}-$ laser pulse, two optical driving fields with a duration of $2 \mu$ s were applied to the $\Lambda$-type three-level system. The SAW field was kept on continuously since it is far from the relevant resonances and contributes only when the corresponding optical driving field is also on. The population in the $m_{s}= \pm 1$ states was detected via the $A_{2}$ transition right after the optical driving pulses.

Figure 5(c) plots the fluorescence from state $A_{2}$ as a function of detuning $\omega_{0}+\omega_{m}-\omega_{ \pm}$, with both $\omega_{0}$ and $\omega_{m}$ fixed. This spectrum directly measures the optically driven, phonon-assisted transitions from the $m_{s}=0$ to

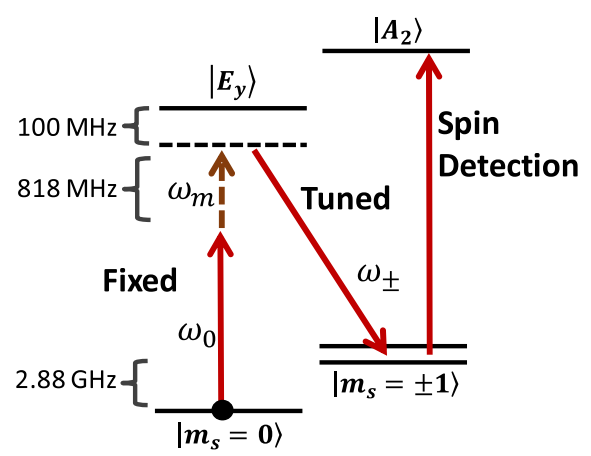

(b)
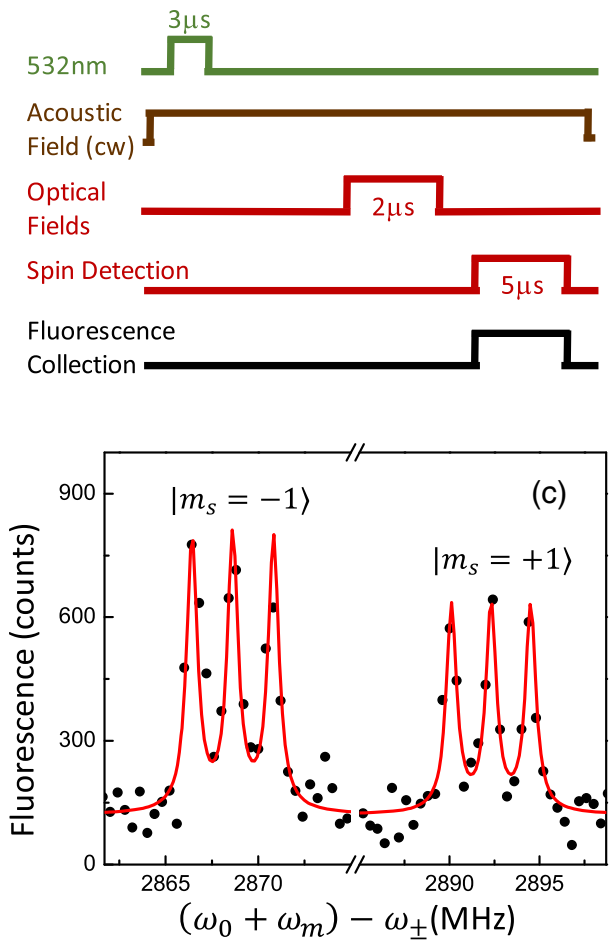

FIG. 5. (a) Energy-level diagram including spin detection used for the sideband spin transition experiments. (b) Pulse sequence used for the spectral-domain experiment. (c) Fluorescence from state $A_{2}$ as a function of $\omega_{0}+\omega_{m}-\omega_{ \pm}$. The solid red line is a fit to six Lorentzians with equal linewidths. A background due to optical pumping has been subtracted from the data.

the $m_{s}= \pm 1$ states. As shown in Fig. 5(c), the sideband spin transitions take place when the Raman resonant condition is satisfied. The spectral linewidth (full width at half maximum) of the transition resonances is $0.7 \mathrm{MHz}$, in agreement with the expected spin dephasing rate. The clearly resolved hyperfine structure of the $m_{s}= \pm 1$ states, with a 2.2-MHz hyperfine splitting, demonstrates that the sideband spin transitions are nuclear-spin selective, thus allowing the use of nuclear spins in quantum acoustics.

\section{B. Time-domain experiment}

To determine the effective Rabi frequency for the sideband spin transitions and to probe effects of the upper-state 
(a)
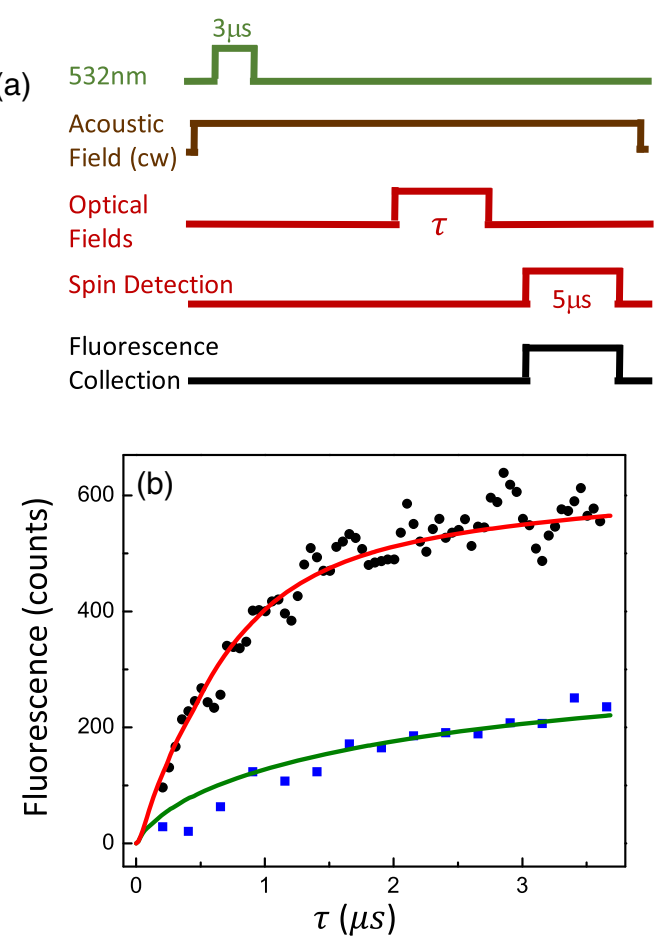

FIG. 6. (a) Pulse sequence used for the transient sideband spin transition experiment. (b) Fluorescence from state $A_{2}$ as a function of the optical pulse duration. Solid circles: $\omega_{0}+\omega_{m}-$ $\omega_{ \pm}$satisfies the Raman resonant condition for the sideband spin transition. Solid squares: $\omega_{0}+\omega_{m}-\omega_{ \pm}$is $6.5 \mathrm{MHz}$ detuned from the Raman resonant condition (the data are smoothed). The solid lines are the theoretical calculations discussed in the text.

excitation, we have carried out transient experiments, in which we measure the spin population in the $m_{s}= \pm 1$ states as a function of the duration of the optical driving fields. As shown in Fig. 6(a), the pulse sequence used is the same as that for the spectral-domain experiments except that the duration of the optical driving fields is now a variable parameter.

The solid circles plotted in Fig. 6(b) show the fluorescence from state $A_{2}$ as a function of the optical pulse duration, where we fixed $\omega_{0}, \omega_{ \pm}$, and $\omega_{m}$ such that the Raman resonant condition for the sideband spin transition was satisfied for the state with $m_{s}=+1$ and $m_{n}=+1$, where $m_{n}$ denotes nitrogen nuclear-spin projection. The initial rise of the fluorescence is primarily due to the sideband spin transition from the $m_{s}=0$ state to the $m_{s}=$ +1 state, with the rise time determined by $\Omega_{s s}$. Optical pumping resulting from the excitation and subsequent decay of the upper state can also lead to population in the $m_{s}=+1$ state. To single out this process, we performed an experiment with the same experimental condition as that used for the solid circles in Fig. 6(b), except that $\left(\omega_{0}+\right.$ $\left.\omega_{m}-\omega_{ \pm}\right) / 2 \pi$ is $6.5 \mathrm{MHz}$ away from the Raman resonant condition. The experimental result is plotted as the solid squares in Fig. 6(b).
The solid lines in Fig. 6(b) show the theoretical calculations based on the density matrix equations, where we have taken $\Omega_{R}=\Omega_{ \pm}$and $\Gamma_{1}$, the decay rate from the $E_{y}$ state to the $m_{s}= \pm 1$ states, as adjustable parameters. The calculations yield $\Gamma_{1} / 2 \pi=1.8 \mathrm{MHz}$ and $\Omega_{R} / 2 \pi=8 \mathrm{MHz}$, which agrees with the Rabi frequency derived from the phonon-assisted $C P T$ experiment using the same optical powers. From these results, we obtain an effective Rabi frequency for the sideband spin transition, $\Omega_{s s} / 2 \pi=$ $0.3 \mathrm{MHz}$. The good agreement between the experiment and theory shows that both the sideband spin transitions and the optical pumping are well characterized by the density matrix equations.

In the limit of large dipole detuning, the upper-state population in the $\Lambda$-type system scales with $1 / \Delta^{2}$. Both the optical pumping rate and the optically induced decoherence rate, $\gamma_{\mathrm{opt}}$, thus also scale with $1 / \Delta^{2}$. In comparison, $\Omega_{s s}$ scales with $1 / \Delta$. In this regard, strong excited-state mediated spin-phonon coupling can be achieved with negligible optical pumping or optically induced decoherence. For example, by setting $\Omega_{ \pm} /|\Delta|$ and $\Omega_{0} /|\Delta|$ to $1 / 60$, we can keep $\gamma_{\text {opt }} / 2 \pi$ to about $1 \mathrm{kHz}$. With $|\Delta|=25 \omega_{m}$ Eq. (4) then yields a single-phonon Rabi frequency $g_{s s}$ that is 3 orders of magnitude greater than what can be achieved with direct ground-state spin-phonon coupling. More optimal excitedstate-mediated spin-phonon coupling with greater $g_{s s}$ and smaller $\gamma_{\text {opt }}$ can be achieved by exploiting techniques such as a shortcut to adiabatic passage [57].

\section{SUMMARY AND OUTLOOK}

In summary, by coupling a SAW to an electron spin in diamond through a $\Lambda$-type three-level system, we have realized both phonon-assisted $C P T$ and optically driven sideband spin transitions. These experiments demonstrate that we can take advantage of the strong excited-state electron-phonon interaction to mediate and control the coupling between spin and mechanical degrees of freedom while avoiding decoherence associated with the excited state. Note that $\Lambda$-type three-level systems with strong excited-state strain coupling provide an excellent experimental platform for exploring spin-based quantum acoustics. Our approach can also be extended to other emerging spin systems with spin defect centers such as $\mathrm{SiC}$ [58], as well as to quantum-dot and superconducting systems. A diamond nanomechanical resonator featuring optically driven sideband spin transitions resembles a trapped-ion system. For a resonator with a modest mechanical Q factor, phonon lasing from a single spin, as well as cooling of the mechanical resonator via coupling to an electron spin, can be explored. A nanomechanical resonator with a sufficiently high mechanical Q factor can enable us to achieve strong spin-phonon coupling at the level of a single phonon and to pursue the highly successful paradigm of trappedion-based quantum computing in a solid-state system. 


\section{ACKNOWLEDGMENTS}

This work is supported by NSF under Grant No. 141 4462 and by AFOSR.

\section{APPENDIX A: THEORETICAL MODEL}

We consider a $\Lambda$-type three-level system, driven by two optical fields and an acoustic field, as shown in Fig. 1(b). The two dipole optical transitions, with frequency $\nu_{1}$ and $\nu_{2}$, couple to the two optical fields, with frequency $\omega_{1}$ and $\omega_{2}$ and Rabi frequency $\Omega_{1}$ and $\Omega_{2}$, respectively. With the rotating wave approximation, the Hamiltonian of the system is given by

$$
\begin{aligned}
H= & \hbar \omega_{m} b^{+} b-\hbar \nu_{1}\left|g_{1}\right\rangle\left\langle g_{1}\left|-\hbar \nu_{2}\right| g_{2}\right\rangle\left\langle g_{2}\left|+\hbar g\left(b^{+}+b\right)\right| e\right\rangle \\
& \times\langle e|+\hbar \frac{\Omega_{1}}{2}\left(e^{-i \omega_{1} t}|e\rangle\left\langle g_{1}\right|+\text { H.c. }\right) \\
& +\hbar \frac{\Omega_{2}}{2}\left(e^{-i \omega_{2} t}|e\rangle\left\langle g_{2}\right|+\text { H.c. }\right)
\end{aligned}
$$

where $b^{+}$and $b$ are the creation and annihilation operators for the acoustic field with frequency $\omega_{m}$ and $g$ is the electron-phonon coupling rate. Applying the SchriefferWolff transformation

$$
U=\exp \left[\frac{g}{\omega_{m}}\left(b^{+}-b\right)|e\rangle\langle e|\right]
$$

to the Hamiltonian gives

$$
\begin{aligned}
\tilde{H}= & \hbar \omega_{m} b^{+} b-\hbar \nu_{1}\left|g_{1}\right\rangle\left\langle g_{1}\left|-\hbar \nu_{2}\right| g_{2}\right\rangle\left\langle g_{2}\left|+\hbar \frac{g^{2}}{\omega_{m}}\right| e\right\rangle \\
& \times\langle e|+\hbar \frac{\Omega_{1}}{2}\left(e^{-i \omega_{1} t+\frac{g}{\omega_{m}}\left(b^{+}-b\right)}|e\rangle\left\langle g_{1}\right|+\text { H.c. }\right) \\
& +\hbar \frac{\Omega_{2}}{2}\left(e^{-i \omega_{2} t+\frac{g}{\omega_{m}}\left(b^{+}-b\right)}|e\rangle\left\langle g_{2}\right|+\text { H.c. }\right)
\end{aligned}
$$

which has the same form as the trapped-ion Hamiltonian [40,59]. Transforming to an interaction picture, we then have

$$
\begin{aligned}
\tilde{H}_{I}= & \hbar \frac{\Omega_{1}}{2}\left(e^{i \Delta_{1} t} e^{-\frac{g}{\omega_{m}}\left(b^{+} e^{i \omega_{m} t}-b e^{-i \omega_{m} t}\right)}|e\rangle\left\langle g_{1}\right|+\text { H.c. }\right) \\
& +\hbar \frac{\Omega_{2}}{2}\left(e^{i \Delta_{2} t} e^{-\frac{g}{\omega_{m}}\left(b^{+} e^{i \omega_{m} t}-b e^{-i \omega_{m} t}\right)}|e\rangle\left\langle g_{2}\right|+\text { H.c. }\right)
\end{aligned}
$$

where $\Delta_{1}=\left(\nu_{1}-g^{2} / \omega_{m}\right)-\omega_{1}$ and $\Delta_{2}=\left(\nu_{2}-g^{2} / \omega_{m}\right)-\omega_{2}$ are the effective detunings of the two optical fields from their respective dipole transitions.

We assume that the $\Omega_{1}$ field is tuned near the red phonon sideband of the $\left|g_{1}\right\rangle$ to $|e\rangle$ transition $\left(\Delta_{1} \approx \omega_{m}\right)$ and the $\Omega_{2}$ field is tuned near the $\left|g_{2}\right\rangle$ to $|e\rangle$ transition $\left(\Delta_{2} \approx 0\right)$. Expanding $\tilde{H}_{I}$ in $g / \omega_{m}$, which can be viewed as an effective Lamb-Dicke parameter for our solid-state system [40], and keeping only the nearly resonant terms, we can approximate the interaction Hamiltonian as

$$
\begin{aligned}
H_{I}= & \hbar \frac{\Omega_{1}}{2} \frac{g}{\omega_{m}}\left(b e^{i\left(\Delta_{1}-\omega_{m}\right) t}|e\rangle\left\langle g_{1}\right|+\text { H.c. }\right)+\hbar \frac{\Omega_{2}}{2}\left(e^{i \Delta_{2} t}|e\rangle\right. \\
& \left.\times\left\langle g_{2}\right|+\text { H.c. }\right)
\end{aligned}
$$

which is similar to a Hamiltonian for a $\Lambda$-type three-level system driven by two optical fields, with effective detuning, $\Delta_{R}=\Delta_{1}-\omega_{m}$ and $\Delta_{2}$, and effective Rabi frequency, $\Omega_{R}=g \sqrt{n} \Omega_{1} / \omega_{m}$ and $\Omega_{2}$, respectively, where $n$ denotes the average phonon number. Note that the Raman resonant condition is $\Delta_{R}=\Delta_{2}$. The above Hamiltonian is also valid for relatively large $\Delta_{R}$ and $\Delta_{2}$, as long as $\Delta_{R} \approx \Delta_{2}$.

With $\Delta_{R}=\Delta_{2}$, the Hamiltonian given by Eq. (A5) features a dark state,

$$
\left|\psi_{d}\right\rangle=\frac{1}{\sqrt{\Omega_{R}^{2}+\Omega_{2}^{2}}}\left(\Omega_{R}\left|g_{2}\right\rangle-\Omega_{2}\left|g_{1}\right\rangle\right)
$$

with $H_{I}\left|\psi_{d}\right\rangle=0$. This dark state is decoupled from state $|e\rangle$, leading to phonon-assisted $C P T$ of the electron in the two lower states.

The equations of motion for the density matrix elements, $\rho_{i j}$, in the rotating frame, which we have used to model the experiments, can also be derived from $H_{I}$ and are given as

$$
\begin{gathered}
\dot{\rho}_{e 1}=-\left(i \Delta_{R}+\gamma\right) \rho_{e 1}+\frac{i \Omega_{R}}{2}\left(\rho_{e e}-\rho_{11}\right)-\frac{i \Omega_{2}}{2} \rho_{21} \\
\dot{\rho}_{e 2}=-\left(i \Delta_{2}+\gamma\right) \rho_{e 2}+\frac{i \Omega_{2}}{2}\left(\rho_{e e}-\rho_{22}\right)-\frac{i \Omega_{R}}{2} \rho_{12} \quad(\mathrm{~A} 7 \mathrm{~b}) \\
\dot{\rho}_{21}=-\left[i\left(\Delta_{R}-\Delta_{2}\right)+\gamma_{s}\right] \rho_{21}+\frac{i \Omega_{R}}{2} \rho_{2 e}-\frac{i \Omega_{2}}{2} \rho_{e 1} \\
\dot{\rho}_{e e}=-\Gamma \rho_{e e}+\left(\frac{i \Omega_{R}}{2} \rho_{e 1}+\text { c.c. }\right)+\left(\frac{i \Omega_{2}}{2} \rho_{e 2}+\text { c.c. }\right)
\end{gathered}
$$

$$
\begin{aligned}
& \dot{\rho}_{11}=\Gamma_{1} \rho_{e e}-\left(\frac{i \Omega_{R}}{2} \rho_{e 1}+\text { c.c. }\right) \\
& \dot{\rho}_{22}=\Gamma_{2} \rho_{e e}-\left(\frac{i \Omega_{2}}{2} \rho_{e 2}+\text { c.c. }\right)
\end{aligned}
$$

where $\gamma_{s}$ and $\gamma$ are the decay rates for the spin coherence and optical dipole coherence, respectively, and $\Gamma=\Gamma_{1}+\Gamma_{2}$ is the total decay rate for the upper-state population, with $\Gamma_{1}$ and $\Gamma_{2}$ being the decay rate to $\left|g_{1}\right\rangle$ and $\left|g_{2}\right\rangle$, respectively. For the theoretical calculation, we have used $\gamma_{s} / 2 \pi=0.35 \mathrm{MHz}$, which is primarily due to spin dephasing induced by the nuclear-spin bath, and $\Gamma / 2 \pi=14 \mathrm{MHz}$, as determined experimentally. We have assumed $\gamma=\Gamma / 2+\gamma_{\text {orb }}$, where $\gamma_{\text {orb }}$ is the dephasing rate due to coupling to the orbital degrees of freedom, and we have taken $\gamma_{\mathrm{orb}} / 2 \pi=12 \mathrm{MHz}$ [56]. We have also used $\Gamma_{1} / 2 \pi=1.8 \mathrm{MHz}$, as derived from the 
optical pumping experiment in Fig. 6(b). NV spectral diffusion is treated as a Gaussian distribution of the optical transition frequency with a linewidth of $140 \mathrm{MHz}$, as derived from the excitation spectrum of the $E_{y}$ resonance.

\section{APPENDIX B: EFFECTS OF STRAIN-INDUCED ELECTRIC FIELDS}

For our experiments, the strain field of a SAW can induce a propagating electric field in the piezoelectric $\mathrm{ZnO}$ layer. This strain-induced electric field can couple to NV centers, though theoretically this coupling is expected to be small compared with the deformation potential coupling. This is because the strain-induced electric field is relatively small [60] and it is mostly confined in the $\mathrm{ZnO}$ layer; in addition, the NV center is about a few microns ( $3 \mu \mathrm{m}$ in our experiment) below the diamond surface. For an experimental confirmation, we rapidly heated up the sample from $8 \mathrm{~K}$ to room temperature. When we cooled the sample back down again to $8 \mathrm{~K}$, the phonon sidebands in the excitation spectrum vanished, but the IDT still functioned, though with a slightly reduced amplitude. In this case, the rapid temperature change broke or significantly weakened the bonds between the $\mathrm{ZnO}$ and diamond. Although the electric field induced by the strain field still propagates in the $\mathrm{ZnO}$ layer, the SAW no longer propagates in diamond. We can only recover the phonon sideband by removing and then redepositing the $\mathrm{ZnO}$ layer. This experiment shows that the coupling between the strain-induced electric field and the $\mathrm{NV}$ center used in our experiment is negligible compared with the deformation potential coupling.

[1] M. V. Gustafsson, T. Aref, A. F. Kockum, M. K. Ekstrom, G. Johansson, and P. Delsing, Propagating Phonons Coupled to an Artificial Atom, Science 346, 207 (2014).

[2] O. O. Soykal, R. Ruskov, and C. Tahan, Sound-Based Analogue of Cavity Quantum Electrodynamics in Silicon, Phys. Rev. Lett. 107, 235502 (2011).

[3] A. Albrecht, A. Retzker, F. Jelezko, and M. B. Plenio, Coupling of Nitrogen Vacancy Centres in Nanodiamonds by Means of Phonons, New J. Phys. 15, 083014 (2013).

[4] S. D. Bennett, N. Y. Yao, J. Otterbach, P. Zoller, P. Rabl, and M. D. Lukin, Phonon-Induced Spin-Spin Interactions in Diamond Nanostructures: Application to Spin Squeezing, Phys. Rev. Lett. 110, 156402 (2013).

[5] H. Wang and G. Burkard, Mechanically Induced Two-Qubit Gates and Maximally Entangled States for Single Electron Spins in a Carbon Nanotube, Phys. Rev. B 92, 195432 (2015).

[6] M. J. A. Schuetz, E. M. Kessler, G. Giedke, L. M. K. Vandersypen, M. D. Lukin, and J. I. Cirac, Universal Quantum Transducers Based on Surface Acoustic Waves, Phys. Rev. X 5, 031031 (2015).

[7] I. Wilson-Rae, P. Zoller, and A. Imamoglu, Laser Cooling of a Nanomechanical Resonator Mode to its Quantum Ground State, Phys. Rev. Lett. 92, 075507 (2004).
[8] K. V. Kepesidis, S. D. Bennett, S. Portolan, M. D. Lukin, and P. Rabl, Phonon Cooling and Lasing with NitrogenVacancy Centers in Diamond, Phys. Rev. B 88, 064105 (2013).

[9] J. M. Pirkkalainen, S. U. Cho, J. Li, G. S. Paraoanu, P. J. Hakonen, and M. A. Sillanpaa, Hybrid Circuit Cavity Quantum Electrodynamics with a Micromechanical Resonator, Nature (London) 494, 211 (2013).

[10] A. D. O'Connell et al., Quantum Ground State and SinglePhonon Control of a Mechanical Resonator, Nature (London) 464, 697 (2010).

[11] J. R. Gell, M. B. Ward, R. J. Young, R. M. Stevenson, P. Atkinson, D. Anderson, G. A. C. Jones, D. A. Ritchie, and A. J. Shields, Modulation of Single Quantum Dot Energy Levels by a Surface-Acoustic-Wave, Appl. Phys. Lett. 93, 081115 (2008).

[12] O. D. D. Couto, S. Lazic, F. Iikawa, J. A. H. Stotz, U. Jahn, R. Hey, and P. V. Santos, Photon Anti-Bunching in Acoustically Pumped Quantum Dots, Nat. Photonics 3, 645 (2009).

[13] M. Metcalfe, S. M. Carr, A. Muller, G. S. Solomon, and J. Lawall, Resolved Sideband Emission of InAs/GaAs Quantum Dots Strained by Surface Acoustic Waves, Phys. Rev. Lett. 105, 037401 (2010).

[14] R. P. G. McNeil, M. Kataoka, C. J. B. Ford, C. H. W. Barnes, D. Anderson, G. A. C. Jones, I. Farrer, and D. A. Ritchie, On-Demand Single-Electron Transfer between Distant Quantum Dots, Nature (London) 477, 439 (2011).

[15] I. Yeo et al., Strain-Mediated Coupling in a Quantum DotMechanical Oscillator Hybrid System, Nat. Nanotechnol. 9, 106 (2014).

[16] F. J. R. Schulein, E. Zallo, P. Atkinson, O. G. Schmidt, R. Trotta, A. Rastelli, A. Wixforth, and H. J. Krenner, Fourier Synthesis of Radiofrequency Nanomechanical Pulses with Different Shapes, Nat. Nanotechnol. 10, 512 (2015).

[17] O. Arcizet, V. Jacques, A. Siria, P. Poncharal, P. Vincent, and S. Seidelin, A Single Nitrogen-Vacancy Defect Coupled to a Nanomechanical Oscillator, Nat. Phys. 7, 879 (2011).

[18] S. Kolkowitz, A. C. B. Jayich, Q. P. Unterreithmeier, S. D. Bennett, P. Rabl, J. G. E. Harris, and M. D. Lukin, Coherent Sensing of a Mechanical Resonator with a Single-Spin Qubit, Science 335, 1603 (2012).

[19] E. R. MacQuarrie, T. A. Gosavi, N. R. Jungwirth, S. A. Bhave, and G. D. Fuchs, Mechanical Spin Control of Nitrogen-Vacancy Centers in Diamond, Phys. Rev. Lett. 111, 227602 (2013).

[20] J. Teissier, A. Barfuss, P. Appel, E. Neu, and P. Maletinsky, Strain Coupling of a Nitrogen-Vacancy Center Spin to a Diamond Mechanical Oscillator, Phys. Rev. Lett. 113, 020503 (2014).

[21] P. Ovartchaiyapong, K. W. Lee, B. A. Myers, and A. C. B. Jayich, Dynamic Strain-Mediated Coupling of a Single Diamond Spin to a Mechanical Resonator, Nat. Commun. 5, 4429 (2014).

[22] E. R. MacQuarrie, T. A. Gosavi, S. A. Bhave, and G. D. Fuchs, Continuous Dynamical Decoupling of a Single Diamond Nitrogen-Vacancy Center Spin with a Mechanical Resonator, Phys. Rev. B 92, 224419 (2015). 
[23] A. Barfuss, J. Teissier, E. Neu, A. Nunnenkamp, and P. Maletinsky, Strong Mechanical Driving of a Single Electron Spin, Nat. Phys. 11, 820 (2015).

[24] E. R. MacQuarrie, T. A. Gosavi, A. M. Moehle, N. R. Jungwirth, S. A. Bhave, and G. D. Fuchs, Coherent Control of a Nitrogen-Vacancy Center Spin Ensemble with a Diamond Mechanical Resonator, Optica 2, 233 (2015).

[25] S. Meesala, Y. I. Sohn, H. A. Atikian, S. Kim, M. J. Burek, J. T. Choy, and M. Loncar, Enhanced Strain Coupling of Nitrogen-Vacancy Spins to Nanoscale Diamond Cantilevers, Phys. Rev. Applied 5, 034010 (2016).

[26] D. D. Awschalom, L. C. Bassett, A. S. Dzurak, E. L. Hu, and J. R. Petta, Quantum Spintronics: Engineering and Manipulating Atom-Like Spins in Semiconductors, Science 339, 1174 (2013).

[27] W. B. Gao, A. Imamoglu, H. Bernien, and R. Hanson, Coherent Manipulation, Measurement and Entanglement of Individual Solid-State Spins Using Optical Fields, Nat. Photonics 9, 363 (2015).

[28] G. Balasubramanian et al., Ultralong Spin Coherence Time in Isotopically Engineered Diamond, Nat. Mater. 8, 383 (2009).

[29] P. C. Maurer et al., Room-Temperature Quantum Bit Memory Exceeding One Second, Science 336, 1283 (2012).

[30] L. Robledo, L. Childress, H. Bernien, B. Hensen, P. F. A. Alkemade, and R. Hanson, High-Fidelity Projective Read-Out of a Solid-State Spin Quantum Register, Nature (London) 477, 574 (2011).

[31] L. Jiang et al., Repetitive Readout of a Single Electronic Spin via Quantum Logic with Nuclear Spin Ancillae, Science 326, 267 (2009).

[32] P. Neumann, J. Beck, M. Steiner, F. Rempp, H. Fedder, P. R. Hemmer, J. Wrachtrup, and F. Jelezko, Single-Shot Readout of a Single Nuclear Spin, Science 329, 542 (2010).

[33] M. V. G. Dutt, L. Childress, L. Jiang, E. Togan, J. Maze, F. Jelezko, A. S. Zibrov, P. R. Hemmer, and M. D. Lukin, Quantum Register Based on Individual Electronic and Nuclear Spin Qubits in Diamond, Science 316, 1312 (2007).

[34] J. R. Maze, A. Gali, E. Togan, Y. Chu, A. Trifonov, E. Kaxiras, and M. D. Lukin, Properties of Nitrogen-Vacancy Centers in Diamond: The Group Theoretic Approach, New J. Phys. 13, 025025 (2011).

[35] M. W. Doherty, N. B. Manson, P. Delaney, and L. C. L. Hollenberg, The Negatively Charged Nitrogen-Vacancy Centre in Diamond: The Electronic Solution, New J. Phys. 13, 025019 (2011).

[36] A. Batalov, V. Jacques, F. Kaiser, P. Siyushev, P. Neumann, L. J. Rogers, R. L. McMurtrie, N. B. Manson, F. Jelezko, and J. Wrachtrup, Low Temperature Studies of the ExcitedState Structure of Negatively Charged Nitrogen-Vacancy Color Centers in Diamond, Phys. Rev. Lett. 102, 195506 (2009).

[37] K. W. Lee, D. Lee, P. Ovartchaiyapong, J. Minguzzi, J. R. Maze, and A. C. B. Jayich, Strain Coupling of a Mechanical Resonator to a Single Quantum Emitter in Diamond, Phys. Rev. Applied 6, 034005 (2016).

[38] D. A. Golter, T. Oo, M. Amezcua, K. A. Stewart, and H. L. Wang, Optomechanical Quantum Control of a
Nitrogen-Vacancy Center in Diamond, Phys. Rev. Lett. 116, 143602 (2016).

[39] E. R. MacQuarrie, M. Otten, S. K. Gray, and G. D. Fuchs, Cooling a Mechanical Resonator with a NitrogenVacancy Center Ensemble Using a Room Temperature Excited State Spin-Strain Interaction, arXiv:1605.07131.

[40] D. Leibfried, R. Blatt, C. Monroe, and D. Wineland, Quantum Dynamics of Single Trapped Ions, Rev. Mod. Phys. 75, 281 (2003).

[41] D. J. Wineland, Nobel Lecture: Superposition, Entanglement, and Raising Schrodinger's Cat, Rev. Mod. Phys. 85, 1103 (2013).

[42] C. Monroe and J. Kim, Scaling the Ion Trap Quantum Processor, Science 339, 1164 (2013).

[43] Y. Tao, J. M. Boss, B. A. Moores, and C. L. Degen, SingleCrystal Diamond Nanomechanical Resonators with Quality Factors Exceeding One Million, Nat. Commun. 5, 3638 (2014).

[44] P. Ovartchaiyapong, L. M. A. Pascal, B. A. Myers, P. Lauria, and A.C. B. Jayich, High Quality Factor Single-Crystal Diamond Mechanical Resonator, Appl. Phys. Lett. 101, 163505 (2012).

[45] M. J. Burek, N. P. de Leon, B. J. Shields, B. J. M. Hausmann, Y. W. Chu, Q. M. Quan, A. S. Zibrov, H. Park, M. D. Lukin, and M. Loncar, Free-Standing Mechanical and Photonic Nanostructures in Single-Crystal Diamond, Nano Lett. 12, 6084 (2012).

[46] P. Rath, S. Ummethala, C. Nebel, and W. H. P. Pernice, Diamond as a Material for Monolithically Integrated Optical and Optomechanical Devices, Phys. Status Solidi A 212, 2385 (2015).

[47] B. Khanaliloo, H. Jayakumar, A. C. Hryciw, D. P. Lake, H. Kaviani, and P. E. Barclay, Single-Crystal Diamond Nanobeam Waveguide Optomechanics, Phys. Rev. X 5, 041051 (2015).

[48] D. A. Golter and H. L. Wang, Optically Driven Rabi Oscillations and Adiabatic Passage of Single Electron Spins in Diamond, Phys. Rev. Lett. 112, 116403 (2014).

[49] D. A. Golter, T. K. Baldwin, and H. L. Wang, Protecting a Solid-State Spin from Decoherence Using Dressed Spin States, Phys. Rev. Lett. 113, 237601 (2014).

[50] H. Nakahata, S. Fujii, K. Higaki, A. Hachigo, H. Kitabayashi, S. Shikata, and N. Fujimori, Diamond-Based Surface Acoustic Wave Devices, Semicond. Sci. Technol. 18, S96 (2003).

[51] C. Santori et al., Coherent Population Trapping of Single Spins in Diamond under Optical Excitation, Phys. Rev. Lett. 97, 247401 (2006).

[52] E. Togan, Y. Chu, A. Imamoglu, and M. D. Lukin, Laser Cooling and Real-Time Measurement of the Nuclear Spin Environment of a Solid-State Qubit, Nature (London) 478, 497 (2011).

[53] C. G. Yale, B. B. Buckley, D. J. Christle, G. Burkard, F. J. Heremans, L. C. Bassett, and D. D. Awschalom, AllOptical Control of a Solid-State Spin Using Coherent Dark States, Proc. Natl. Acad. Sci. U.S.A. 110, 7595 (2013).

[54] M.O. Scully and M.S. Zubairy, Quantum Optics (Cambridge University Press, Cambridge, 1997). 
[55] K. Bergmann, N. V. Vitanov, and B. W. Shore, Perspective: Stimulated Raman Adiabatic Passage: The Status after 25 Years, J. Chem. Phys. 142, 170901 (2015).

[56] B. B. Zhou, A. Baksic, H. Ribeiro, C. G. Yale, F. J. Heremans, P. C. Jerger, A. Auer, G. Burkard, A. A. Clerk, and D. D. Awschalom, Accelerated Quantum Control Using Superadiabatic Dynamics in a Solid-State Lambda System, arXiv:1607.06503.

[57] X. Chen, I. Lizuain, A. Ruschhaupt, D. Guery-Odelin, and J. G. Muga, Shortcut to Adiabatic Passage in Two- and
Three-Level Atoms, Phys. Rev. Lett. 105, 123003 (2010).

[58] W. F. Koehl, B. B. Buckley, F. J. Heremans, G. Calusine, and D. D. Awschalom, Room Temperature Coherent Control of Defect Spin Qubits in Silicon Carbide, Nature (London) 479, 84 (2011).

[59] F. Mintert and C. Wunderlich, Ion-Trap Quantum Logic Using Long-Wavelength Radiation, Phys. Rev. Lett. 87, 257904 (2001).

[60] P. Y. Yu and M. Cardona, Fundamentals of Semiconductors (Springer-Verlag, Berlin, 1996). 